\title{
MODELAGEM DO EQUILÍBRIO DE FASES ENTRE ÉSTERES DE ÁCIDOS GRAXOS DE ÓLEO DE PEIXE COM DIÓXIDO DE CARBONO SUPERCRÍTICO.
}

\author{
M. P. SILVA ${ }^{1}$, A.L. GONÇALVES ${ }^{1}$, J. V. M. LOPES $^{2}$, N. T. MACHADO ${ }^{2}$ \\ M. E. ARAÚJO \\ ${ }^{1}$ Universidade Federal do Pará, Programa de Pós-Graduação em Engenharia Química \\ ${ }^{2}$ Universidade Federal do Pará, Faculdade de Engenharia Química, Laboratório de \\ Simulação de Processos e Termodinâmica Aplicada \\ E-mail para contato:meaaraujo@gmail.com
}

\begin{abstract}
RESUMO -As equações de estado são os modelos mais utilizados para o cálculo do equilíbrio de fases a altas pressões. Este trabalho teve como objetivo a avaliação da capacidade de predição do equilíbrio de fases entre ésteres de ácidos graxos $/ \mathrm{CO}_{2}$ de óleo de peixe, das equações de Soave-Redlich-Kwong e PengRobinson com as regras de mistura de van der Waals (quadrática), Mathias-KlotzPrausnitz e das equações de Soave-Redlich-Kwong e Peng-Robinson modificadas com as regras de mistura de Kwak-Mansoori. Os parâmetros de interação binária foram determinados empregando os programas Edeflash e PE. Ambas as equações apresentaram precisão na correlação dos dados experimentais demonstrando que as regras de mistura mostraram-se adequadas para a descrição do equilíbrio dos sistemas estudados.
\end{abstract}

\section{INTRODUÇÃO}

O processamento em escala industrial de produtos da pesca gera uma grande quantidade de óleo de peixe de descarte. A composição majoritária dos óleos de peixe são triglicerídeos os quais são formados por diferentes cadeias de ácidos graxos. Os mais importantes ácidos graxos poliinsaturados ( $\omega 3)$ de cadeia longa presentes nos óleos de peixe são o eicosapentanóico,

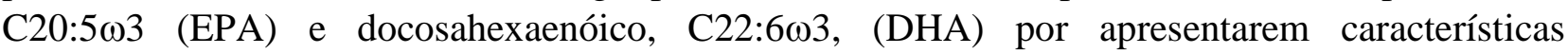
nutraceuticas para aplicações em alimentos funcionais, suplementos dietéticos e fármacos (Devi et al., 2008).

A tecnologia com $\mathrm{CO}_{2}$ supercrítico em colunas de multiestágios em contracorrente apresenta-se como um processo importante para o fracionamento desses subprodutos da indústria por ser conduzida em condições mais suaves, evitando a degradação térmica principalmente dos ácidos graxos insaturados. Os estudos experimentais descritos na literatura utilizam para aumentar a concentração de EPA e DHA, a corrente de alimentação na forma de ésteres de ácidos graxos de óleo de peixe, principalmente como ésteres etílicos (FOEE) (Maschietti e Pedacchia, 2014, Gironi e Maschietti, 2006, Riha e Brunner, 1999, 2000). A simulação desses processos requer informações sobre o equilíbrio de fases dos compostos envolvidos.

As equações de estado são os modelos mais utilizados para o cálculo do equilíbrio de fases a altas pressões. Dados experimentais de ésteres de ácidos graxos de óleo de peixe em $\mathrm{CO}_{2}$ 
supercrítico são escassos. Bharath et al. (1989) mediram dados de equilíbrio líquido-vapor de cinco sistemas binários envolvendo ácidos graxos $/ \mathrm{CO}_{2}$ e ésteres de ácidos graxos $/ \mathrm{CO}_{2}$ de óleo de peixe, e correlacionaram os dados experimentais com a equação de Peng-Robinson com a regra de mistura quadrática de van der Waals com dois parâmetros de interação binária. Chang et al. (2005), apresentaram dados experimentais de equilíbrio para quatro misturas binárias de ésteres dentre eles os ésteres etílicos dos ácidos eicosapentanóico, eC20:5 33 (EPAEE) e docosahexaenóico, $\mathrm{C} 22: 6 \omega 3$ (DHAEE) com $\mathrm{CO}_{2}$ supercrítico. Os dados foram correlacionados com as equações de estado de Peng-Robinson e Soave-Redlich-Kwong com as regras de mistura quadrática com dois parâmetros de interação, Panagiotopoulos-Reid e Adachi-Sugie com três parâmetros de interação.

Este trabalho teve como objetivo a avaliação da capacidade de predição do equilíbrio de fases entre ésteres de ácidos graxos de óleo de peixe com $\mathrm{CO}_{2}$ supercrítico, das equações de Soave-Redlich-Kwong (SRK) e Peng-Robinson (PR) com as regras de mistura de van der Waals (quadrática) com dois parâmetros de interação binária e a regra de mistura de Mathias-KlotzPrausnitz (MKP) com três e com dois parâmetros de interação binária e, das equações de SoaveRedlich-Kwong (SRK) e Peng-Robinson (PR) modificadas com a regra de mistura com três parâmetros de interação de Kwak-Mansoori (KM1).

\section{MÉTODOS E PROCEDIMENTOS}

As equações de estado e as regras de mistura utilizadas para a modelagem termodinâmica dos sistemas binários, realizada com os programas PE (Pfohl et al, 2000) e Edeflash (Araújo, et $a l .$, 2006) estão apresentadas na Tabela 1. Os parâmetros $a, b$ e $d$ são determinados a partir das propriedades termofísicas das substâncias puras $\left(T_{c}, P_{c} e \omega\right)$, com as interações entre os componentes da mistura representadas pelos parâmetros de interação binária.

Para a determinação dos parâmetros de interação binária os programas utilizam o procedimento PT flash e o método matemático Simplex modificado de Nelder e Mead (1965) para a minimização da seguinte função objetivo (FO):

$$
F O=\sqrt{\frac{1}{n} \sum_{i=1}^{n}\left(\frac{x_{i}^{e}-x_{i}^{c}}{x_{i}^{e}}\right)^{2}+\left(\frac{y_{i}^{e}-y_{i}^{c}}{y_{i}^{e}}\right)^{2}}
$$

Para a correlação de dados foram utilizadas as propriedades críticas $(P c, T c)$, e os fatores acêntricos $(\omega)$ publicadas pelos autores dos dados experimentais. Na Tabela 2 são apresentados os valores das propriedades termofísicas dos compostos puros utilizados neste trabalho. Na Tabela 3 estão descritas as fontes dos dados experimentais de equilíbrio líquido-vapor dos sistemas binários da literatura correlacionados neste trabalho. 
Tabela 1-Equações de estado e regras de mistura

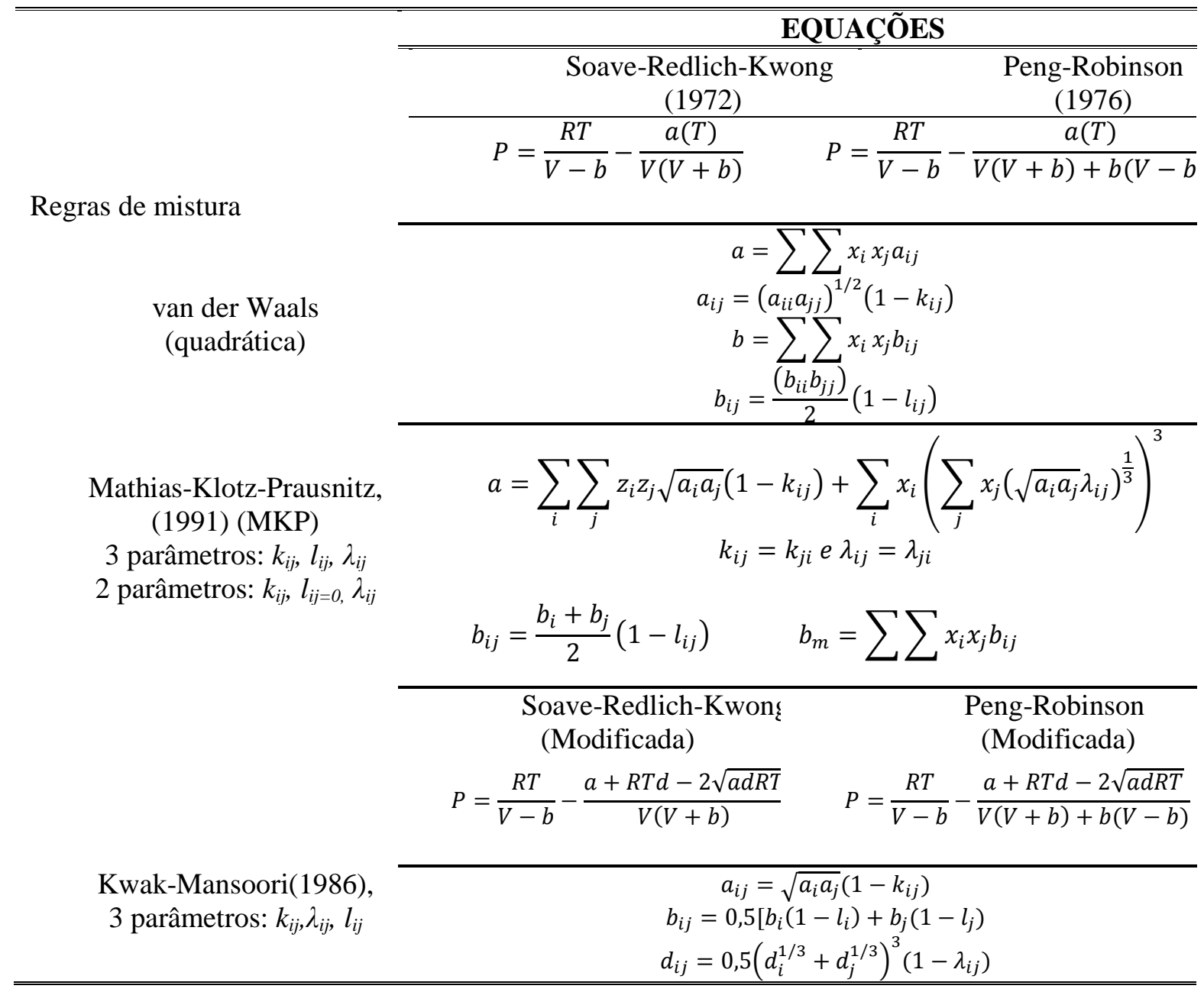

Tabela 2 - Propriedades físicas das substâncias puras

\begin{tabular}{llllllc}
\hline \hline Substâncias & $C A S$ & $\mathrm{M}(\mathrm{g} / \mathrm{mol})$ & $\mathrm{Tb}(\mathrm{K})$ & $\mathrm{Tc}(\mathrm{K})$ & $\mathrm{Pc}(\mathrm{bar})$ & $\omega$ \\
\hline \hline $\mathrm{CO}_{2}$ & $124-38-9$ & 44 & - & 304,1 & 73,8 & 0,225 \\
$\mathrm{e} 18-0$ & $111-61-5$ & 312,53 & 594,3 & 761,8 & 16,17 & 0,887 \\
$\mathrm{e} 18-1 \omega 9$ & $111-62-6$ & 310,5 & 631,4 & 782 & 12,10 & 0,992 \\
$\mathrm{e} 18-2 \omega 6$ & $544-35-4$ & 308,5 & 630,8 & 783,7 & 12,64 & 1,082 \\
$\mathrm{~m} 18-1 \omega 9$ & $112-62-9$ & 296,5 & 700,7 & 875,31 & 11,22 & 1,0797 \\
$\mathrm{~m} 18-2 \omega 6$ & $112-63-0$ & 94,5 & 696,5 & 866,9 & 11,62 & 0,9835 \\
EPAEE$(\mathrm{e} 20-5 \omega 3)$ & $94-70-2$ & 302,46 & 671,5 & 833,8 & 11,81 & 1,013 \\
DHAEE$(\mathrm{e} 22-6 \omega 3)$ & $73310-11-9$ & 356,5 & 702,5 & 867,1 & 11,02 & 0,990 \\
\hline \hline
\end{tabular}


Tabela 3- Fonte dos dados experimentais de equilíbrio dos sistemas binários

\begin{tabular}{|c|c|c|c|c|}
\hline Sistemas $\mathrm{CO}_{2}+$ & Faixa de $\mathrm{T}(\mathrm{K})$ & $\mathrm{P}$ (bar) & Pontos. Exp & Referências \\
\hline e18-0 & $313,15-333,15$ & $14,7-182,6$ & $9-11$ & \multirow{5}{*}{$\begin{array}{c}\text { Bharath } \\
\text { et al. (1989) }\end{array}$} \\
\hline e18-1 $\omega 9$ & $313,15-333,15$ & $11,4-186,2$ & $9-16$ & \\
\hline 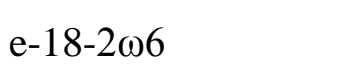 & $313,15-333,15$ & $19,7-169-7$ & $8-13$ & \\
\hline EPAEE (e20-5 23$)$ & $313,15-333,15$ & $20-200$ & 7 & \\
\hline 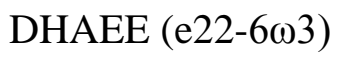 & $313,15-333,15$ & $18,7-210,7$ & $4-8$ & \\
\hline $\mathrm{m} 18-1 \omega 9$ & $313-333$ & $28,6-180,3$ & $12-13$ & \multirow{4}{*}{$\begin{array}{l}\text { Chang } \\
\text { et al. (2005) }\end{array}$} \\
\hline $\mathrm{m} 18-2 \omega 6$ & $313-333$ & $28,6-180,3$ & $12-14$ & \\
\hline EPAEE $(\mathrm{e} 20-5 \omega 3)$ & $313-333$ & $28,6-207,9$ & $13-14$ & \\
\hline 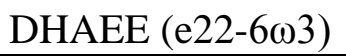 & $313-333$ & $42,4-235,4$ & $13-14$ & \\
\hline
\end{tabular}

\section{RESULTADOS E DISCUSSÕES}

Na Tabela 4 estão apresentados os valores dos parâmetros de interação binária, as FO e os valores dos erros médios absolutos $(\Delta \mathrm{x}$ e $\Delta \mathrm{y})$ determinados pelo programa Edeflash, empregando as equações de SRK e PR modificadas com a regra de mistura Kwak-Mansoori (KM1). Na Tabela 5 estão apresentados os valores dos parâmetros de interação binária, as FO e os valores dos erros médios absolutos $(\Delta \mathrm{x}$ e $\Delta \mathrm{y})$ determinados pelo programa $\mathrm{PE}$, empregando as equações de SRK e PR combinadas com a regra de mistura de van der Waals quadrática com dois parâmetros de interação, com a regra de mistura Mathias-Klotz-Prausnitz com dois e três parâmetros.

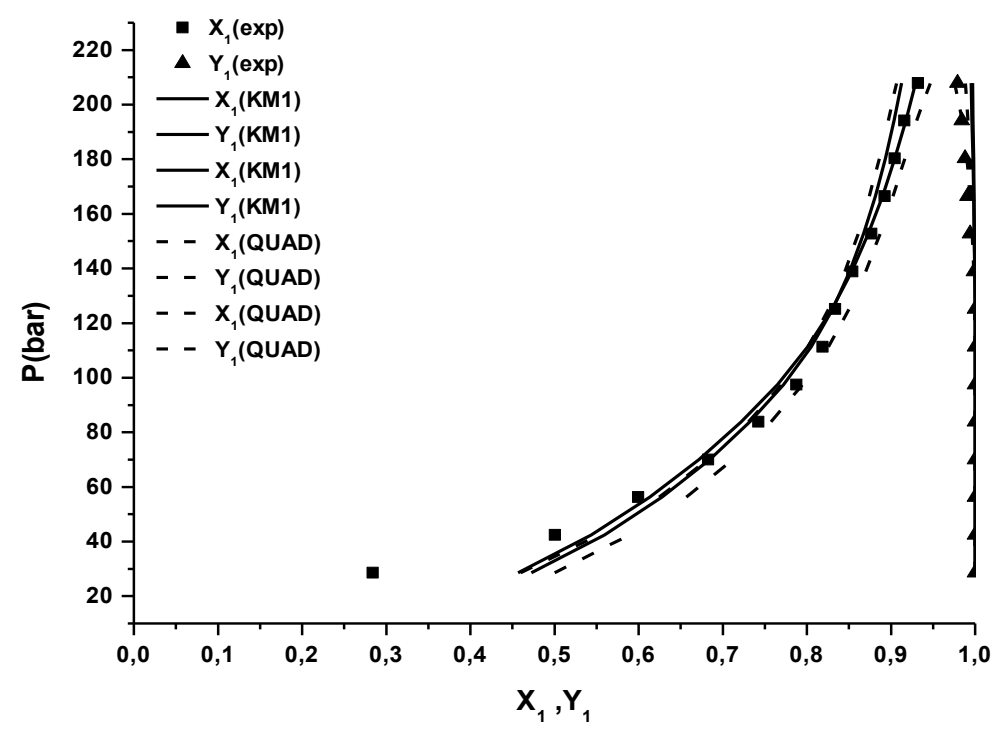

Figura 1 - Equilíbrio líquido-vapor para o sistema EPAEE/ $\mathrm{CO}_{2}$ a $333 \mathrm{~K}$. 
Tabela 4 - Parâmetros de interação binária e os valores dos erros médios absolutos ( $\Delta \mathrm{x}$ e $\Delta \mathrm{y})$ para as equações SRK e PR modificadas com a regra de mistura KM1 utilizando o programa Edeflash

\begin{tabular}{|c|c|c|c|c|c|c|c|c|c|c|c|c|c|}
\hline \multirow{2}{*}{ Sistemas $\mathrm{CO}_{2}+$} & \multicolumn{7}{|c|}{$\begin{array}{c}\text { Soave-Redlich-Kwong } \\
\text { KM1 }\end{array}$} & \multicolumn{6}{|c|}{$\begin{array}{c}\text { Peng-Robinson } \\
\text { KM1 }\end{array}$} \\
\hline & $\mathrm{T}(\mathrm{K})$ & $\bar{k}_{i j}$ & $l_{i j}$ & $\bar{\lambda}_{i j}$ & $F O$ & $\Delta x \%$ & $\Delta y \%$ & $k_{i j}$ & $l_{i j}$ & $\lambda_{i j}$ & $F O$ & $\Delta x \%$ & $\Delta y \%$ \\
\hline \multirow{3}{*}{ e18-0 } & 313,15 & $-0,19824$ & $-0,38236$ & 0,12124 & 1,77 & 1,90 & 0,05 & $-0,19624$ & $-0,38236$ & 0,12053 & 1,54 & 1,81 & 0,06 \\
\hline & 323,15 & $-0,20373$ & $-0,35168$ & 0,12188 & 0,98 & 2,93 & 0,03 & $-0,20193$ & $-0,36955$ & 0,12086 & 0,79 & 3,02 & 0,03 \\
\hline & 333,15 & $-0,21010$ & $-0,34049$ & 0,12271 & 1,68 & 2,27 & 0,05 & $-0,20770$ & $-0,35931$ & 0,12177 & 1,40 & 2,53 & 0,04 \\
\hline \multirow{3}{*}{ 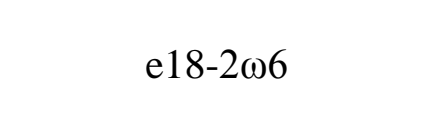 } & 313,15 & $-0,09358$ & $-0,33901$ & 0,12521 & 28,15 & 38,4 & 0,35 & $-0,07798$ & $-0,33901$ & 0,12522 & 28,13 & 35,18 & 0,35 \\
\hline & 323,15 & $-0,11028$ & $-0,35015$ & 0,12227 & 29,56 & 38,29 & 0,49 & $-0,33014$ & $-0,51475$ & 0,11039 & 3,71 & 4,50 & 0,36 \\
\hline & 333,15 & $-0,34632$ & $-0,50802$ & 0,11101 & 5,06 & 5,10 & 0,17 & $-0,33999$ & $-0,53677$ & 0,11062 & 4,54 & 5,83 & 0,15 \\
\hline \multirow{2}{*}{$\mathrm{m} 18-1 \omega 9$} & 313 & $-0,32695$ & $-0,49996$ & 0,11294 & 9,79 & 2,47 & 0,24 & $-0,32254$ & $-0,51060$ & 0,11211 & 9,68 & 1,58 & 0,25 \\
\hline & 333 & $-0,34219$ & $-0,47199$ & 0,11478 & 10,44 & 1,69 & 0,42 & $-0,33486$ & $-0,46517$ & 0,11466 & 10,64 & 1,01 & 0,45 \\
\hline \multirow{2}{*}{$\mathrm{m} 18-2 \omega 6$} & 313 & $-0,32486$ & $-0,50991$ & 0,11241 & 9,99 & 3,56 & 0,24 & $-0,31945$ & $-0,51332$ & 0,11200 & 9,71 & 2,08 & 0,25 \\
\hline & 333 & $-0,34046$ & $-0,47414$ & 0,11460 & 10,50 & 1,91 & 0,42 & $-0,33463$ & $-0,44201$ & 0,11599 & 10,71 & 1,23 & 0,46 \\
\hline \multirow{3}{*}{ EPAEE (Chang et al.) } & 313 & $-0,28562$ & $-0,35970$ & 0,12098 & 12,37 & 2,74 & 0,42 & $-0,27398$ & $-0,36306$ & 0,12086 & 13,21 & 3,48 & 0,44 \\
\hline & 333 & $-0,30823$ & $-0,39340$ & 0,11893 & 10,82 & 2,26 & 0,36 & $-0,29670$ & $-0,38688$ & 0,11945 & 11,56 & 2,76 & 0,39 \\
\hline & 313,15 & $-0,30713$ & $-0,53374$ & 0,10953 & 2,86 & 4,25 & 0,45 & $-0,30574$ & $-0,56913$ & $-0,30574$ & 2,98 & 4,55 & 0,52 \\
\hline \multirow[t]{2}{*}{ EPAEE (Bharath et al.) } & 323,15 & $-0,31268$ & $-0,52461$ & 0,11087 & 2,00 & 4,04 & 0,09 & $-0,30975$ & $-0,55378$ & $-0,30975$ & 1,70 & 3,66 & 0,12 \\
\hline & 333,15 & $-0,31804$ & $-0,50369$ & 0,11124 & 1,07 & 3,04 & 0,10 & $-0,31311$ & $-0,54604$ & 0,10942 & 0,87 & 2,78 & 0,13 \\
\hline \multirow{3}{*}{ DHAEE (Chang et al.) } & 313 & $-0,34048$ & $-0,42467$ & 0,11735 & 9,77 & 1,36 & 0,42 & $-0,33229$ & $-0,41176$ & 0,11808 & 10,57 & 2,01 & 0,45 \\
\hline & 333 & $-0,34139$ & $-0,38778$ & 0,11872 & 11,32 & 2,51 & 0,36 & $-0,32868$ & $-0,37493$ & 0,11954 & 11,13 & 2,03 & 0,37 \\
\hline & 313,15 & $-0,31895$ & $-0,35813$ & 0,12114 & 7,80 & 3,37 & 0,51 & $-0,35977$ & $-0,47222$ & 0,11512 & 5,57 & 8,09 & 0,17 \\
\hline \multirow[t]{2}{*}{ DHAEE (Bharath et al.) } & 323,15 & $-0,36513$ & $-0,52203$ & 0,11182 & 2,20 & 7,82 & 0,08 & $-0,35251$ & $-0,61779$ & 0,10662 & 1,51 & 4,73 & 0,19 \\
\hline & 333,15 & $-0,36526$ & $-0,58598$ & 0,10641 & 0,83 & 4,62 & 0,10 & $-0,35908$ & $-0,63045$ & 0,10401 & 0,84 & 4,06 & 0,14 \\
\hline
\end{tabular}


Tabela 5 - Erros médios absolutos determinados pelo programa PE, empregando as equações de estado SRK e PR, combinadas com as regras de mistura quadrática, MKP3 e MKP2

\begin{tabular}{|c|c|c|c|c|c|c|c|c|c|c|c|c|c|}
\hline \multirow{3}{*}{ Sistemas $\mathrm{CO}_{2}+$} & \multicolumn{7}{|c|}{ Soave-Redlich-Kwong } & \multicolumn{6}{|c|}{ Peng-Robinson } \\
\hline & \multirow[t]{2}{*}{$\mathrm{T}(\mathrm{K})$} & \multicolumn{2}{|c|}{ QUAD } & \multicolumn{2}{|c|}{ MKP3 } & \multicolumn{2}{|c|}{ MKP2 } & \multicolumn{2}{|c|}{ QUAD } & \multicolumn{2}{|c|}{ MKP3 } & \multicolumn{2}{|c|}{ MKP2 } \\
\hline & & $\Delta x \%$ & $\Delta y \%$ & $\Delta x \%$ & $\Delta y \%$ & $\Delta y \%$ & $\Delta y \%$ & $\Delta x \%$ & $\Delta y \%$ & $\Delta x \%$ & $\Delta y \%$ & $\Delta x \%$ & $\Delta y \%$ \\
\hline \multirow{4}{*}{ e18-0 } & 313,15 & 4,75 & 0,03 & 14,89 & 0,20 & 2,67 & 0,03 & 3,17 & 0,17 & 0,66 & 0,003 & 19,58 & 0,21 \\
\hline & 323,15 & 4,27 & 0,13 & 2,99 & 0,13 & 5,22 & 0,14 & 3,81 & 0,14 & 3,22 & 0,14 & 6,59 & 0,17 \\
\hline & 333,15 & 5,78 & 0,12 & 3,01 & 0,11 & 5,84 & 0,11 & 2,99 & 0,12 & 4,99 & 0,12 & 8,51 & 0,13 \\
\hline & 313,15 & 1,65 & 0,07 & 1,73 & 0,08 & 3,75 & 0,18 & 2,86 & 0,09 & 3,23 & 0,09 & 6,41 & 0,21 \\
\hline \multirow{3}{*}{ e18-1 109} & 323,15 & 5,20 & 0,19 & 9,06 & 0,33 & 8,23 & 0,32 & 1,98 & 0,19 & 3,74 & 0,21 & 6,76 & 0,22 \\
\hline & 333,15 & 2,98 & 0,16 & 1,81 & 0,21 & 3,58 & 0,19 & 1,73 & 0,20 & 3,44 & 0,19 & 4,19 & 0,16 \\
\hline & 313,15 & 3,08 & 0,07 & 2,71 & 0,07 & 1,83 & 0,14 & 4,47 & 0,09 & 4,80 & 0,09 & 5,05 & 0,11 \\
\hline \multirow{2}{*}{ 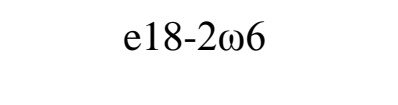 } & 323,15 & 1,69 & 0,16 & 5,30 & 0,19 & 5,35 & 0,19 & 5,61 & 0,21 & 5,52 & 0,21 & 6,36 & 0,21 \\
\hline & 333,15 & 1,66 & 0,18 & 3,18 & 0,13 & 2,93 & 0,12 & 7,17 & 0,14 & 5,59 & 0,14 & 7,35 & 0,14 \\
\hline $\mathrm{m} 18-1 \omega 9$ & 333 & 4,97 & 0,24 & 4,06 & 0,21 & 3,81 & 0,21 & 2,91 & 0,11 & 6,17 & 0,24 & 6,22 & 0,25 \\
\hline $\mathrm{m} 18-2 \omega 6$ & 333 & 2,59 & 0,17 & 2,91 & 0,19 & 2,66 & 0,18 & 3,09 & 0,18 & 3,47 & 0,11 & 4,84 & 0,22 \\
\hline \multirow{3}{*}{ EPAEE(Chang et al.) } & 313 & 1,83 & 0,19 & 0,59 & 0,27 & 4,42 & 0,23 & 3,81 & 0,24 & 1,33 & 0,21 & 1,71 & 0,69 \\
\hline & 333 & 2,95 & 0,22 & 1,86 & 0,08 & 2,46 & 0,05 & 3,66 & 0,09 & 2,99 & 0,12 & 2,54 & 0,09 \\
\hline & 313,15 & 2,44 & 0,11 & 0,49 & 0,13 & 0,60 & 0,23 & 0,55 & 0,16 & 0,74 & 0,18 & 9,13 & 0,77 \\
\hline \multirow{2}{*}{ EPAEE(Bharath et al.) } & 323,15 & 1,02 & 0,05 & 0,44 & 0,03 & 0,85 & 0,04 & 0,76 & 0,02 & 0,77 & 0,03 & 0,59 & 0,02 \\
\hline & 333,15 & 0,77 & 0,06 & 1,68 & 0,04 & 0,63 & 0,06 & 0,45 & 0,03 & 0,36 & 0,02 & 0,29 & 0,02 \\
\hline \multirow{2}{*}{ DHAEE (Chang et al.) } & 313 & 2,76 & 0,19 & 1,60 & 0,64 & 1,03 & 0,66 & 1,50 & 0,71 & 2,80 & 0,33 & 4,12 & 0,19 \\
\hline & 333 & 1,62 & 0,17 & 2,48 & 0,13 & 4,35 & 0,16 & 1,64 & 0,39 & 1,31 & 0,19 & 1,69 & 0,23 \\
\hline
\end{tabular}


A Figura 1 apresenta os dados experimentais de equilíbrio líquido-vapor de Chang et. al. (2005) para o sistema EPAEE/ $\mathrm{CO}_{2}$ a $333 \mathrm{~K}$ comparados com os valores preditos pelas equação de SRK com a regra de mistura quadrática com o programa PE e pela equação de SRK modificada com a regra de mistura Kwak-Mansoori (KM1) com o programa Edeflash.

$\mathrm{Na}$ Tabela 6 estão apresentados os valores calculados dos desvios médios absolutos $(\Delta x \%$, $\Delta y \%$ ), para os sistemas EPAEE/ $\mathrm{CO}_{2}$ e DHAEE/ $\mathrm{CO}_{2}$ publicados por Chang et. al. (2005) e os valores determinados neste trabalho, com a equação de estado Soave-Redlich-Kwong com a regra de mistura quadrática, empregando o programa PE. Vale destacar que Chang et. al. (2005) utilizou a função objetivo soma dos erros absolutos de ambas as fases, enquanto que neste trabalho foi utilizada a função objetivo soma dos quadrados dos erros relativos de ambas as fases.

Tabela 5. -Comparação dos erros médios absolutos determinados pelo programa PE, empregando a equação de SRK, com a regra de mistura quadrática.

\begin{tabular}{lccccc}
\hline \hline & & \multicolumn{3}{c}{ Soave-Redlich-Kwong } \\
\cline { 3 - 6 } Sistemas $\mathrm{CO}_{2+}$ & & \multicolumn{2}{c}{ Chang et al.(2005) } & \multicolumn{3}{c}{ Este trabalho } \\
\cline { 3 - 6 } & $\mathrm{T}(\mathrm{K})$ & \multicolumn{4}{c}{ QUADRÁTICA } \\
\cline { 3 - 6 } EPAEE & 313 & 1,193 & 0,188 & 1,83 & 0,19 \\
& 333 & 4,076 & 0,117 & 2,95 & 0,22 \\
DHAEE & 313 & 3,113 & 0,087 & 2,76 & 0,19 \\
& 333 & 1,275 & 0,11 & 1,62 & 0,17 \\
\hline \hline
\end{tabular}

\section{CONCLUSÕES}

Este trabalho apresentou uma revisão de dados experimentais de equilíbrio líquido-vapor da literatura de sistemas binários entre ésteres de ácidos graxos $/ \mathrm{CO}_{2}$ de óleo de peixe, e os correlacionou com as equações de estado cúbicas de Soave-Redlich-Kwong e Peng-Robinson com as regras de mistura de van der Waals (quadrática), com dois parâmetros de interação binária e a regra de mistura de Mathias-Klotz-Prausnitz (MKP) com três e com dois parâmetros de interação binária e, com as equações de Soave-Redlich-Kwong (SRK) e Peng-Robinson (PR) modificadas com a regra de mistura com três parâmetros de interação de Kwak-Mansoori (KM1).

Os resultados mostraram que o uso das regras de mistura quadrática, MKP com dois parâmetros, MKP com três parâmetros e KM1 com três parâmetros de interação, descreveram quantitativamente os dados experimentais, não apresentando diferenças significativas em relação aos erros médios absolutos calculados.

\section{REFERÊNCIAS}

ARAÚJO, M. E.; AZEVEDO-JR., C. M.; SANTOS, J. L.; PENA, M. V.; MEIRELES, M. A. A. Programa Computacional para o Cálculo do Equilíbrio de Fases de Sistemas 
Multicomponentes empregando o Algoritmo Flash. Proceedings of XXVII Iberian Latin American Congress on Computational Methods in Engineering, CD-ROM, p. 1-19, setembro, 2006.

BHARATH R., INOMATA H., ARAI K., SHOJI K., NOGUCHI Y. Vapor-Liquid Equilibria for Binary Mixtures of Carbon Dioxide and Fatty Acid Ethyl Esters. Fluid Phase Equilibria, v. 50, p.315-327, 1989.

CHANG, C. J.; LEE, M.; LI, B.; CHEN, P. Vapor-liquid equilibria and densities of CO2 with four unsaturated fatty acid esters at elevated pressures. Fluid Phase Equilibria, v.233, p.56-65, 2005 .

DEVI, P.; ZHANG, H.; DAMSTRUP, M.L.; GUO, Z.; ZHANG, L.; LUE, B.M.; XU, X. Enzymatic synthesis of designer lipids. OCL, v. 15, n. 3, p. 189-195, 2008.

GIRONI, F.; MASCHIETTI, M. Separation of fish oils ethyl esters by means of supercritical carbon dioxide:Thermodynamic analysis and process modeling. Chemical Engineering Science, v.61, p.5114-5126, 2006.

KWAK, T. Y., MANSOORI, G. A. Van der Waals Mixing Rules for CubicEquations of State. Applications for Supercritical Fluid Extraction Modeling. Chemical. Engineering.Science.,v. 41, p. 1303, 1986.

MASCHIETTI, M.; PEDACCHIA, A. Supercritical carbon dioxide separation of fish oil ethyl esters by means of a continuous countercurrent process with an internal reflux. The Journal of Supercritical Fluids, v.86, p.76-84, 2014.

MATHIAS, P.M.; KLOTZ, H.C.; PRAUSNITZ, J.M. Equation-of-State Mixing Rules for Multicomponent Mixtures: The Problem of Invariance. Fluid Phase Equilibria.v. 67, p. 31, 1991.

NELDER, J. A.; MEAD, R. A Simplex Method for Function Minimization. Computer Journal. v. 7, pp. 308-313, 1965.

PENG, D.Y.; ROBINSON, D.B. A New Two-Constant Equation of State. Industrial andEngineering Chemical Fundamental.v.15, p.59-64, 1976.

PFOHL, O.; PETKOV, S.; BRUNER, G. PE 200: A Powerful Tool to Correlate Phase Equlibria; Herbert Utz: Munich, 2000; ISBN 3-89675-751-2.

RIHA, V.; BRUNNER, G. Phase equilibrium of fish ethyl esters with supercritical carbon dioxide. The Journal of Supercritical Fluids, v.15, p.33-50, 1999.

RIHA, V.; BRUNNER, G. Separation of fish oil ethyl esters with supercritical carbon dioxide. The Journal of Supercritical Fluids, v.17, p.55-64, 2000.

SOAVE, G. Equilibrium Constants from a Modified Redlich-Kwong Equation of State. Chemical Engineering Science.v.27, p. 1192-1203, 1972. 